\title{
Las relaciones sociales y su papel en la motivación hacia la práctica de actividad física en adolescentes: Un enfoque cualitativo Social relatedness and its role in adolescents' motivation towards physical activity: a qualitative approach \\ Jorge Lizandra, Carmen Peiró-Velert \\ Universidad de Valencia (España)
}

\begin{abstract}
Resumen. El presente estudio adopta un enfoque cualitativo para profundizar en el papel que juegan las relaciones sociales en la motivación hacia la práctica de actividad física (AF) de los adolescentes españoles. Para ello se desarrolló un estudio de casos a partir del análisis de contenido de las entrevistas a seis adolescentes españoles (tres chicas y tres chicos), provenientes de distintas regiones y habitantes tanto de contextos urbanos como rurales. Dicho análisis interpretativo permitió la obtención inductiva de cuatro principales categorías del conjunto total de datos. Los resultados, presentados mediante un relato realista muestran que, a lo largo de la adolescencia, la familia deja de ser un referente social clave en favor del grupo de iguales. Además, estas relaciones con el grupo social surgen, unas veces, como importantes mediadoras que facilitan conductas motivadas hacia la AF, y otras aparecen como inhibidoras de esas conductas, dependiendo del tipo de relación y actividades que se desarrollen. Por último, emerge una presión de la cultura social para que las y los adolescentes asuman conductas más propias de las personas adultas, como son las conductas sedentarias o alejadas de la práctica de AF regular. Se puede concluir que, aunque las relaciones sociales de los y las adolescentes pueden facilitar conductas que motiven hacia la AF, también pueden llegar a dificultarlas, por lo que resulta conveniente crear entornos en los que se favorezcan relaciones sociales satisfactorias y alejadas de conductas inactivas y nocivas para la salud.
\end{abstract}

Palabras clave: actividad física, adolescentes, relaciones sociales, motivación, investigación cualitativa.

Abstract. This study adopts a qualitative approach to deepen the role played by social relationships in the motivation towards the practice of physical activity (PA) in Spanish adolescents. A case study was developed based on the content analysis of the interviews of six Spanish adolescents (three girls and three boys), from different regions and both urban and rural contexts. This interpretative analysis allowed to inductively obtain four main categories of the total data set. The results, presented through a realistic report, show that during adolescence family ceases to be a key social reference in favour of peers. In addition, these relationships with the social group sometimes arise as important mediators facilitating motivational behaviours towards PA, whereas in other occasion they act as inhibitors of these behaviours, depending on the type of relationship and activities developed by the group. Finally, pressure from the social culture emerges for adolescents to assume behaviours more typical in adults, such as sedentary behaviours or in general far from the practice of regular PA. Although adolescents' social relationships can facilitate behaviours that motivate PA, they can also become an issue, so it is convenient to create environments in which satisfactory social relationships are favoured and away from inactive and harmful behaviours for health.

Keywords: physical activity, adolescents, social relationships, motivation, qualitative research.

\section{Introducción}

La motivación hacia la práctica de la actividad física (AF) es un tema ampliamente estudiado tanto desde el ámbito de la psicología general como de la psicología del deporte en particular (Biddle \& Wang, 2003; De Andrade, Salguero, González-Boto \& Márquez, 2006; Haerens, Kirk, Cardon, De Bourdeaudhuij \& Vansteenkiste, 2010). Además, existe un interés especial por el estudio de la motivación en la etapa de la adolescencia por tratarse de un momento del ciclo vital caracterizado por la sucesión de numerosos cambios a nivel social y cognitivo que contribuyen a la consolidación de hábitos de vida que probablemente permanecerán en la edad adulta (Menschik, Ahmed, Alexander \& Blum, 2008). Precisamente uno de los cambios de conducta que más preocupan es la disminución gradual del tiempo de AF que, a pesar de ser conocidos sus beneficios tanto a nivel físico, psicológico como social (Carazo-Vargas y Moncada-Jiménez, 2015), difícilmente alcanzan a cumplir los niveles recomendados para mantener y mejorar la salud de los y las jóvenes (Abarca-Sos, Zaragoza, Generelo \& Julián, 2010; Oviedo et al., 2013).

\footnotetext{
Fecha recepción: 01-02-189. Fecha de aceptación: 25-06-19

Jorge Lizandra

jorge.lizandra@uv.es
}

Al parecer una de las claves para la adherencia a la práctica de AF durante la adolescencia se encuentra en ayudar a que encuentren el disfrute en ella, de manera que ese sentimiento de satisfacción facilite que perseveren en la conducta. Del mismo modo, mantener una buena forma física e imagen corporal, así como relacionarse con otras personas, se incluyen entre los motivos para realizar AF (MECD, 2015). En ese sentido, la asignatura de educación física (EF) debería ser un espacio proclive para proporcionar estas experiencias (Álvarez, López, Gómez, Mesa, \& Martínez, 2017). No obstante, no hallar tal disfrute o bien encontrarlo en otro tipo de actividades de ocio más sedentario (Devís-Devís, BeltránCarrillo \& Peiró-Velertt, 2015), puede estar detrás de la progresiva disminución del tiempo de AF. De hecho, la falta de interés, la preferencia por actividades que requieran menor esfuerzo incluso la pereza, se han declarado en estudios previos como causas para el abandono de la práctica de AF (Epstein \& Roemmich, 2001; MECD, 2015).

Tradicionalmente, el estudio de la motivación se fundamenta en macro-teorías generales del comportamiento humano como la Teoría de la Autodeterminación (TAD) (Deci \& Ryan, 1985) o la Teoría de Metas de Logro (Nicholls, 1989). La primera postula que la conducta tenderá a estar más intrínsecamente motivada en la medida en que se satisfagan las necesidades psicológicas básicas de la persona de sentirse competente (competencia), sentirse autónomo en sus 
decisiones (autonomía) y sentirse relacionado y parte de un grupo (relación). La teoría de metas de logro, por su parte, destaca que en el estado motivacional y el bienestar de las personas influyen tanto variables disposicionales (orientación al ego o la tarea), como variables situacionales (percepción de los climas sociales).

En ese sentido, Ntoumanis (2001) afirma que, en los climas de implicación a la tarea, las conductas de cooperación entre compañeros son habituales, mientras que en un clima de implicación hacia el ego es más común que se fomente la competitividad entre los miembros del grupo. Estas afirmaciones, orientadas a situaciones de práctica de AF, conducen a pensar que en los entornos en los que se valore el esfuerzo y la implicación en la tarea, por encima del resultado, en los que se refuerce la colaboración sobre la competición, especialmente en contextos en los que se den niveles de habilidad heterogéneos, será más fácil evocar experiencias que satisfagan las necesidades psicológicas básicas de los adolescentes. De hecho, parece que los climas orientados a la tarea también predicen positivamente la relación con los demás, así como su percepción de competencia, hasta el punto de facilitar que puedan llegar a estados psicológicos óptimos que les lleven a persistir en su actividad (GonzálezCutre, Sicilia, Moreno \& Fernández-Balboa, 2009). Por tanto, ambas teorías confluyen en la importancia que tiene para las personas desarrollar su actividad en un entorno que favorezca la satisfacción de sus necesidades y sus logros.

No obstante, aunque la literatura muestra suficientes evidencias en las que se dan estas relaciones más lineales, otros estudios muestran cómo sobre el constructo de la motivación puede influir un amplio espectro de elementos, tanto de carácter interno como externo que, al no darse habitualmente de forma aislada, dotan de una mayor complejidad a las teorías motivacionales (García-Bengoechea \& Strean, 2007). De hecho, la propia definición de clima introducida por Ames y Archer (1988) aduce a la diversidad de entornos sociales que pueden influir en la toma de decisiones de las personas. Tanto es así que las experiencias previas vividas en la escuela y fuera de ella, las interacciones con la familia y su entorno y las que se producen en las actividades que realizan en su tiempo de ocio, son determinantes en la intención de seguir realizando práctica física en el presente y en el futuro (Green, Smith \& Roberts, 2005).

Por otro lado, cabe indicar que muchas de las investigaciones que muestran un mayor alineamiento con los postulados de las teorías motivacionales clásicas utilizan predominantemente un enfoque metodológico cuantitativo (Biddle, Cury, Goudas, Sarrazin, Famose \& Durand, 1995). En cambio, en los últimos años han ido proliferando varios estudios cualitativos que evidencian, no solo la cantidad de personas del entorno social del adolescente que influyen en la toma de sus decisiones (padres, madres, entrenadores/as, amistades), sino que aluden precisamente a la multifactorialidad de causas que moderan la actitud y la motivación del/la adolescente hacia la actividad física y el deporte (Gillison, Osborn, Standage \& Skevington, 2009; Hassandra, Goudas \& Chroni, 2003; Keegan, Harwood, Spray \& Lavallee, 2009).

Por tanto, si se entiende la motivación como un proceso psico-socio-afectivo complejo que mueve a la acción (Moreno, Otero, Crowe, Sullivan \& Rausch, 2013), son diversas las variables sociales, ambientales e individuales que confluyen y determinan no solo la elección de una tarea concreta, sino también la intensidad con la que se ejecutará, así como la persistencia en la misma. En ese sentido, en la literatura parece haber consenso en que el nivel de competencia es uno de los elementos más determinantes para que los jóvenes persistan en una determinada tarea. No obstante, recientes estudios destacan la importancia de las relaciones sociales que establecen con sus iguales como uno de los elementos clave en la motivación (Méndez-Giménez, Cecchini-Estrada \& Fernández-Río, 2018), fenómeno que coincide además con el desarraigo progresivo de la significatividad social de los padres y las madres, al menos en el contexto de la AF (Fernández, Contreras, García \& González, 2010; Li, Iannotti, Haynie, Perlus \& Simons-Morton, 2014). Prueba de ello sería el estudio de Stuntz y Weiss (2009), cuyos resultados mostraron que aquellos adolescentes que formaban parte consolidada de un determinado grupo deportivo estaban más motivados intrínsecamente, se sentían más competentes y se mostraban más convencidos de continuar con su AF.

En este sentido, conocer las variables que influyen en la decisión de realizar o no AF es una de las necesidades emergentes en el área, sobre todo cuando los datos de distintos estudios epidemiológicos apuntan hacia el abandono progresivo de esta durante la adolescencia (Abraldes \& Argudo, 2009), a pesar de que sus beneficios sean ampliamente conocidos (Gebremariam et al., 2012; McKercher et al., 2014; Ntoumanis \& Standage, 2009). El propósito de este estudio, de corte cualitativo, es explorar la relevancia que han tenido las relaciones sociales en los motivos de práctica de AF durante la adolescencia a partir de sus reflexiones recogidas mediante entrevistas semiestructuradas.

\section{Metodología}

\section{Muestra}

La muestra proviene de una primera fase de estudio cuantitativo sobre estilos de vida activos en el que participaron adolescentes de seis zonas geográficas de España (Norte, Nordeste, Este, Centro, Sur e Islas Canarias). Fue en una segunda fase de carácter cualitativo en la que se realizaron 26 entrevistas. Para el presente estudio se eligieron como informantes a 6 estudiantes de ESO y Bachillerato, tres chicos y tres chicas con edades comprendidas entre los 14 y 18 años, siendo todos nacidos en España y procedentes de tres de las zonas anteriormente indicadas. La selección se realizó atendiendo al criterio de variedad sustancial (Patton, 1990). Concretamente, dos chicos provienen de la zona centro y uno de la zona nordeste, siendo 2 de ellos de un entorno urbano y uno de los de la zona centro residente en una zona rural. Con respecto a las chicas, dos de ellas provienen de la zona este y una de ellas de la zona centro, siendo una de las chicas de la zona este de entorno rural y las otras dos de entorno urbano. Todos ellos y ellas tienen como característica común el mostrar un perfil de baja participación en AF, mientras que el comportamiento sedentario variaba desde alto a moderado o bajo.

Procedimiento, fuente de recogida de datos y consideraciones éticas

Antes de iniciar el trabajo de campo de la primera fase cuantitativa, se hizo acopio de las autorizaciones, tanto de 
las familias como de los centros educativos en los que se realizó. En uno de los ítems del cuestionario se les solicitó un primer consentimiento para participar en la fase de entrevistas. En el caso de que accedieran, indicaban un teléfono de contacto. Fue así como se contactó con los y las participantes con las que se convino una cita para realizar las entrevistas. En esta conversación telefónica se entabló contacto también con el padre, la madre o tutor legal, para informarles del propósito de la entrevista, garantizándoles mantener el anonimato y que pudieran abandonar la investigación en cualquier momento, si lo deseaban. Además, en el caso de los menores de edad, se obtuvo un segundo consentimiento de los familiares. La recogida de datos se realizó mediante una entrevista semiestructurada, de una duración aproximada de una hora y media y se llevó a cabo, previa cita, en la residencia particular de cada informante. La entrevista se inició solicitando al/a la informante un relato sobre sus primeros recuerdos y vivencias realizando AF en general, o actividades deportivas concretas. Asimismo, se disponía de un guion con posibles preguntas que ayudaran a enfocar y profundizar en el tema de estudio. Para preservar en la privacidad, se han utilizado pseudónimos en el informe.

\section{Estrategias de investigación}

Las estrategias de investigación seguidas en este trabajo son de carácter cualitativo. Concretamente se optó por un estudio de casos pues, según Stake (2007), los casos son de interés tanto en el área de educación como en otras áreas sociales, siendo en su mayoría constituidos por personas que se asemejan en cierta forma, pero que a la vez son únicos. De ahí que los informantes se consideren valiosos, tanto por aquello que tienen en común, como por sus características subjetivas.

Parte de las bondades de este tipo de investigación figuran en la habilidad de la persona investigadora de describir y desarrollar los fenómenos para que sean entendidos tanto desde la perspectiva investigadora como del lector/a. De ahí que parte del método de investigación utilizado se apoye en el concepto de reflexibilidad, que se define como el proceso interpretativo mediante el que el investigador se convierte en actor de su estudio e instrumento de la indagación (Janesick, 2000). La reflexividad contribuye a la validez del proceso cualitativo en la medida en la que la persona investigadora es capaz de dejar atrás los juicios a priori o ideas preconcebidas, aspirando a cumplir una función vehicular para obtener datos y comprender la experiencia del otro (Denzin \& Lincoln, 1994). Esta posición del investigador necesariamente produce un espacio para la subjetividad, en el que la experiencia compartida del investigador y los participantes en el estudio permite descubrir el significado de las historias que ocurren a las personas. Poniendo un ejemplo, extraído precisamente de uno de los informantes de este estudio, «si te hablan de la historia de cómo era el pueblo, no es lo mismo lo que te dice un libro, que lo que te dicen aquellos que lo han vivido» [Pedro] (pseudónimo).

\section{Análisis de los datos}

El análisis de datos puede definirse como el trabajo que se realiza con la información pura, natural para fragmentarla y organizarla (Patton, 1990). Las entrevistas fueron grabadas y posteriormente transcritas a formato Word para poder trabajar con ellas y ser analizadas. El siguiente paso consistió en identificar, mediante la lectura y triangulación de la información contenida en las entrevistas, diferentes conceptos o unidades textuales en los que se encontraba puntos de unión y que, a través de la agrupación y clasificación por unidades temáticas, fueron dotándose de sentido, siguiendo los planteamientos de Strauss y Corbin (2002). Seguidamente, se llevó a cabo un proceso de inducción analítica que, como indican autores tales como Goetz y LeCompte (1988) o Martínez, (2006), consistió en la obtención de las categorías del conjunto total de datos. Para ello, los grupos de unidades textuales se fueron asociando en torno a las categorías que surgían de la interpretación de los datos ofrecidos por los estudiantes y que se observan en la tabla 1 . Por tanto, no se partió de un esquema previo de categorías para el estudio, sino que estas surgieron del examen de la información procedente de los ensayos entregados. Cabe indicar que la credibilidad del estudio descansa en la comprobación de las transcripciones de las entrevistas y la actividad de contraste y crítica realizada por el equipo de investigación durante todo el proceso. En último lugar, teniendo organizada la información, se optó por un planteamiento de resultados basado en el relato realista (Sparkes \& Devís, 2007), en el que se han ido incorporando referencias a algunas citas textuales de las entrevistas que sirven como refuerzo para el conjunto de reflexiones e interpretaciones a continuación se exponen.

\begin{tabular}{|c|c|}
\hline Categorías & Códigos \\
\hline Cambio de referentes sociales & $\begin{array}{l}\text {-Padres como referentes en la infancia. } \\
\text {-Grupo de iguales como referente actual } \\
\text {-Padres asumen un nuevo rol en fase actual }\end{array}$ \\
\hline Facilitadores de la actividad física & $\begin{array}{l}\text {-Nivel de competencia } \\
\text {-Relaciones saludables con los iguales } \\
\text {-Determinadas temporadas en entorno rural }\end{array}$ \\
\hline Inhibidores de actividad física & $\begin{array}{l}\text {-Cambio de hábitos sociales } \\
\text {-Relaciones tóxicas con grupo de iguales } \\
\text {-Tendencia a conductas sedentarias }\end{array}$ \\
\hline Influencia de la cultura social & $\begin{array}{l}\text {-Adolescencia como rito social de paso } \\
\text {-Distorsión social del significado de "crecer" } \\
\text {-Influencia de los modelos educativos }\end{array}$ \\
\hline
\end{tabular}

\section{Resultados}

Este estudio trata de explorar en qué medida la multidimensionalidad de las relaciones sociales moderan la actitud y motivación hacia la actividad física y el deporte de seis adolescentes españoles. De los análisis de los datos procedentes de las seis entrevistas surgen cuatro categorías que se han convertido en tres epígrafes con el fin de organizar y secuenciar los resultados y la posterior discusión de estos.

Practicar actividad física... ¿pero con quién?: cesión del protagonismo de la familia al de amigos y amigas.

A medida que los adolescentes van consolidando sus relaciones sociales conforme avanza su edad, la familia deja de ser tan determinante en favor del grupo de iguales que se erige como el elemento socializador más importante. Con relación a la práctica de AF, dejan progresivamente de realizar actividades con los padres, dado que ya no les reporta la satisfacción que anteriormente les producía. Se sienten desubicados en un entorno afectivo en el que ya no encajan y lo sustituyen por otro más afín a sus intereses y necesidades afectivas, como son la relación con su círculo de amista- 
des, independientemente que realicen o no AF.

«Sí, sí que las hacemos [actividades con sus padres], pero a mí ya no me llama la atención. [... Es que me aburro... me aburro con ellos [...] Antes yo me iba con ellos y me lo pasaba bien. Ahora me voy con ellos y digo «y ¿qué pinto yo aquí?» me voy con mis amigas que me lo paso mejor [...] Yo con mis amigas soy de una manera y con mis padres tengo que ser de otra.» [Manuela]

«Hombre a la playa sí que voy con ellos, pero...normalmente voy más con las amigas que con ellos». [María]

«(en el pueblo) íbamos bajo a la calle, cogíamos un balón y jugábamos con el balón, también como aquí hacen hogueras, pues también nos íbamos a la hoguera, también jugábamos, yo que sé a pillar. Cuando jugabas al escondite, sí estaba bastante bien» [María]

Incluso aquellos que, además de mostrar un perfil de baja AF se muestran especialmente sedentarios, al practicar AF en grupo, se animan a hacerlo:

«(los amigos) que te animan a jugar. Pues porque si somos pocos pues no te animas, pero si sois muchos pues dicen: Vamos a jugar a esto. Y tú: venga va...» [Carlos]

«La experiencia más positiva es cuando juego un partido de fútbol con mis amigos. Eso sí que me encanta, me gusta bastante. Como somos amigos de toda la vida, pues me gusta eso» [David]

De aliado a potencial enemigo en la consolidación de hábitos saludables: la importancia del entorno y las relaciones sociales

A la luz de los comentarios de nuestros/as informantes, la transcendencia de las relaciones con los demás ha sido un elemento tan determinante como el nivel de competencia a la hora de implicarse en actividades físico-deportivas.

Así pues, parece que, en el contexto escolar, ambas percepciones (competencia y buenas relaciones sociales) convergen y se influyen mutuamente. En este sentido, los compañeros/as pueden actuar de forma consciente o inconsciente como jueces de la capacidad, (in)competencia motriz, de los adolescentes y partir de su valoración para aceptarlo en el grupo o, por el contrario, ignorarlo o, peor aún, ser blanco de insultos y desprecios. Es decir, la valoración del grupo de iguales de la (in)competencia en el entorno escolar puede llegar a influir negativamente, especialmente en los recreos. En cambio, cuando la relación se da fuera del entorno escolar, los amigos/as pueden jugar un papel más de refuerzo, incluso de modelado:

«Cuando solíamos jugar a futbol (en el recreo) salía muy escarmentado. Me daban balonazos en la cabeza o en la espalda o donde fuera. Siempre acababa mal. [...] normalmente acababa de portero. Los porteros eran los más «porricos» (inhábiles)» [Pedro]

«En verano, ... mi mejor amigo tiene una piscina en su casa. Yo a la municipal llevo tiempo sin ir porque es un caos. Entonces si voy a casa de mi amigo (contexto informal) pues jugamos con una pelota dentro del agua, como si fuera waterpolo» [Pedro]

«Todos los niños se ponen a jugar a fútbol (en el recreo) y te acabas poniendo, te acaba gustando. No sé, acabas probando y poco a poco te vas haciendo un poco al deporte [...] Y luego, pues claro, me enteré del equipo que había. Mis compañeros de clase que jugaban en el equipo. Me apunté. Me gustó bastante, la verdad». [Carlos]

«También muchas veces dicen: "iVenga, vamos a la Fuente del Sabugo! [espacio de su localidad]», que no está muy lejos de aquí. Dicen: «iVamos!, ... y nos sentamos allí al sol y no sé qué», y se traen un balón y con dos piedras hacemos una portería y a jugar a fútbol ahí, todo el día». [Manuela]

Otro aspecto que destacar es la importancia que los chicos que viven en entornos rurales le confieren a la interacción social, como elemento favorecedor de la implicación en la práctica de AF. El hecho de que sean lugares donde apenas hay niños o niñas de su edad supone, en algunos casos, que ese proceso de socialización relacionado con la AF haya sido tardío o haya estado rodeado de mayores dificultades:

«Ahora cuando viene el verano, viene más gente de [la ciudad], al pueblo y, por ejemplo: «iVamos a echar un partido de fútbol!» «iVenga, va!». Ahora casi todos los días estamos echando un partido [...] Pues porque si somos pocos, pues no te animas, pero si somos muchos, pues dicen: « Vamos a jugar a esto!» y tú: «iVenga va!». [David]

«He vivido entre [pueblo1] y [pueblo2] hasta que se murió mi abuelo, que yo tenía 8 años. A no ser que lloviera o hiciera mucho frío, íbamos a andar al campo y lo que se hacía en el colegio [de AF]. Pero, claro, yo era el único chico del pueblo. Hasta que no tuve 6-7 años no había niños, así, con los que salir». [Pedro]

No obstante, esa fuerza del entorno social puede llegar a ser tanto un aliado como un enemigo en la consolidación de la práctica de AF, ya que el factor de relación resulta ser tan determinante para afianzar el hábito de práctica como para el abandono de este hábito y la incorporación, e incluso sustitución, por hábitos poco saludables. Es en estas edades, cuando se empieza a tener contacto con actividades de ocio como salir por la noche, beber y fumar, entre otros hábitos que, además de ser perjudiciales para su salud, a corto y largo plazo, alejan a los y las jóvenes de otras costumbres entendidas como más saludables.

«Pues no sé...en vez de jugar con los amigos en el parque, pues igual te ibas a un bar a echarte una cerveza, recreativos. Sí, siempre te vuelves con la edad un poco más sedentario, dejas. ..menos juego y más. ..Principalmente con 12-13 años que es cuando empiezas a juntarte con gente» [Pedro]

«Con mis amigas yo hago cosas que con mis padres no puedo hacer. Ahora mismo yo con mis amigas digo muchos tacos. Y yo soy muy mala y la estoy liando todos los días. $Y$ luego cogemos y nos compramos una cerveza y un paquete de tabaco y nos vamos a fumar y beber. [Manuela]

La transcendencia social de las palabras. La influencia de la cultura social.

Uno de los resultados más sorprendentes a priori se encuentra en el aparente significado social que tiene el paso de la educación primaria hacia la educación secundaria. La mayoría de las y los informantes comentan que en primaria jugaban tanto en clase de EF como en el recreo, pero que al pasar a la educación secundaria abandonan el juego y adoptan el que parece estar considerado como el comportamiento 
esperado por el nuevo entorno social. Parece que culturalmente jugar sea una cosa de niños y niñas y no de adolescentes, hasta el punto de que algunos de ellos se avergüencen de hacerlo:

«Cuando empiezas en el instituto te lo ves como más grande y dices pues esto [el juego] ya hay que dejarlo...Porque eso es más de pequeños y yo ya estoy en secundaria...me sabe mal decir esto, pero sí». [Pedro]

«... te sientas [en el patio] y ya está... es que esos juegos parece como si fueras más pequeño, entonces al querer aparentar ser más mayor, pues [en el instituto] ya no juegas» [María]

«Hombre en las horas de recreo, de siempre, comer el bocadillo [risas] y hablar...AF...poca, por no decir nada» [David]

"[Al llegar al instituto]...pues ya paras. Porque como allí, se supone que te has hecho más mayor ya no juegas a los juegos, te sientas y ya está. Hombre, había gente que sí, que entrabas y aún jugaban a pillar y eso [...] Es que esos juegos parece que... es como si fueras más pequeño, entonces al querer aparentar ser más mayor, pues ya no juegas. [Manuela]

Por tanto, parece que el hecho de llegar a la educación secundaria se convierte en una especie de rito de paso, socialmente aceptado, que lamentablemente contribuye al descenso en el tiempo de práctica de AF durante la adolescencia.

\section{Discusión}

Aunque se observa que la familia sigue formando parte de la vida de los adolescentes, pues los padres siguen siendo un elemento importante tanto en la vida de sus hijos como en la toma de decisiones (Li y et al., 2014), se aprecia cómo la filiación con su grupo de iguales comienza a consolidarse como elemento de mayor significatividad en esta etapa de sus vidas. Por otro lado, los adolescentes viven en una sociedad en continuo cambio, cuyos estímulos condicionan tanto sus acciones, como sus expectativas, actitudes y valores. Esto significa que el estilo de vida no es el resultado únicamente de un comportamiento individual, sino que es parte de una continua interacción social que siguiendo a Bourdieu (1998), se construye y reconstruye a partir de la interacción con los distintos elementos de la sociedad, de los distintos hábitos adquiridos, así como de las propias apreciaciones y reflexiones que se suceden a lo largo del periodo vital de las personas.

En ese sentido, cabe decir que, aunque es cierto que las necesidades psicológicas básicas, como el sentimiento de competencia, es un importante mediador de la conducta motivacional hacia la práctica de AF (Menéndez \& FernándezRío, 2017; Ntoumanis, 2001), la relación y el reconocimiento de los iguales se postula como un aspecto tanto o más relevante, resultado que se alinea con el estudio de MéndezGiménez, Cecchini-Estrada y Fernández-Río (2018). Este posicionamiento es avalado por otras teorías influyentes en la motivación como la teoría de metas sociales (Moreno \& Conde, 2012). No obstante, también se ha visto que la transcendencia de las relaciones con sus iguales significativos puede resultar ser tanto un aliado como una amenaza para la práctica de la AF. Un ejemplo del efecto positivo del refuerzo social se observa en que incluso en personas con perfil de baja $\mathrm{AF}$, los momentos de encuentro con amigos de su edad suponen un estímulo que les motiva a salir a jugar a diario (Huertas, Martínez, Tercedor \& Delgado, 2010). En cambio, resultados de este trabajo también muestran cómo el tipo de actividades preponderantes en el grupo social en el que el adolescente se inserta o bien del que anhela formar parte, pueden orientarle hacia optar por otro tipo de conductas. Urdan y Maehr (1995) indican que el tipo de valores u objetivos que tenga el grupo social con el que se mantiene una determinada relación, influye directamente sobre los objetivos de cada uno de los integrantes de ese grupo. Por tanto, si el grupo social está alejado de la práctica de AF, es probable que los individuos disminuyan o abandonen la AF en búsqueda de su aprobación social.

El modelo jerárquico de aproximación-evitación propuesto por Elliot y Church (1997) aborda la teoría de las metas sociales desde patrones de comportamiento complejos que podrían ofrecer una explicación al posible doble efecto que se ha visto que puede tener la relación con los iguales. Concretamente, este modelo no solo estudia los aspectos que favorecen a una determinada acción, sino también aquellas razones por las que las personas evitan o se alejan de una conducta (Elliot, Gable \& Mapes, 2006). Teniendo en cuenta que para los adolescentes es tan importante lograr el reconocimiento social, como evitar el rechazo, es muy probable que estas metas sociales puedan estar detrás de la disminución o el abandono de la práctica de AF (Macarro, Romero \& Torres, 2010). Además, el abandono de la AF durante la adolescencia suele llevar asociado la elección de otras actividades de ocio pasivo, que en el peor de los casos puede conducir a hábitos poco saludables e incluso nocivos (Valdemoros, Ponce, Sanz \& Ramos, 2007).

Finalmente, otros motivos para el abandono podrían estar detrás de un discurso social asumido, aceptado y que, por tanto, a priori no genera ningún problema. Detrás de frases alentadas por el/la docente como «ahora ya no estáis en primaria, ahora sois mayores»y las que las familias refuerzan con insinuaciones como «ahora debes comportarte como un mayor», así como en los discursos de la falta de tiempo o incluso de la pereza de una sociedad desmotivada (MECD, 2015), también conducen a veces de manera inconsciente a la reducción o al abandono de la AF. Mensajes de este tipo en una fase sensible para la consolidación de la identidad, como es la adolescencia, pueden llevar a las y los estudiantes a integrar que eso es lo que se espera de ellos en ese contexto social y asumir que tienen que imitar comportamientos adultos. Por tanto, si el referente adulto más cercano e influyente hasta ese momento son los padres y estos mantienen una actitud predominantemente sedentaria y el cambio de entorno escolar destila referentes (alumnado de cursos superiores, profesorado) también sedentarios, la probabilidad de que los y las adolescentes interioricen que ese es el comportamiento esperado será muy elevada.

El contexto educativo de alguna manera también es cómplice del discurso de la adolescencia como rito de paso. El hecho de que la educación secundaria se imparta en los antiguos centros de bachillerato favorece la consolidación de ese discurso. Bolívar (2004) indica que la Educación Se- 
cundaria Obligatoria (E.S.O), desde la aparición de la LOGSE en 1990, anda en busca de una identidad que, a pesar de que la esencia de las leyes educativas precedentes planteen modelos participativos más próximos a las prácticas ya consolidadas en educación primaria, la organización de los centros o las dinámicas sociales se asemeja más a un modelo cercano al bachillerato, aspectos que se ven claramente acrecentados por el hecho de que la E.S.O se imparta en los antiguos institutos de bachillerato. En cambio, podría aprovecharse ese instaurado rito de paso para ofrecer a los y las jóvenes propuestas saludables vinculadas a la práctica de AF dentro de los centros de enseñanza secundaria. Ahora bien, las propuestas no pueden reproducir las actividades que se ofertan o practican en primaria (p. ej. juegos a la hora del patio), sino que deben ofrecer programas que incorporen actividades que se adapten a los intereses y gustos de los adolescentes. Propuestas como la de la Conselleria de Educació de la Comunitat Valenciana con los proyectos deporte, actividad física y salud (PEAFS), que promuevan la práctica de AF fuera del horario lectivo, pero que a su vez están integrados en el proyecto educativo del centro, podrían servir de ejemplo (DOGV, 2017).

\section{Conclusiones}

Este estudio explora el papel que juegan las relaciones sociales en la toma de decisiones de los adolescentes en lo concerniente a la práctica de AF. Se aprecia en las entrevistas como el grupo de iguales sustituye a las familias como referente de socialización deportiva. Además, uno de los resultados más relevantes es que estas relaciones con los iguales durante la adolescencia pueden mediar tanto como facilitadoras e inhibidoras de su (no)implicación en la práctica de AF. Este hecho encuentra una posible explicación en la importancia de las metas sociales y el reconocimiento por el grupo de iguales para los adolescentes, que además puede desempeñar roles distintos tanto dentro como fuera del contexto escolar, hipótesis sobre las que convendría seguir investigando.

Otro resultado a tener en cuenta es el 'rito de paso' que conduce al cambio desde un rol 'activo' aceptado en la etapa educativa de primaria a un rol 'inactivo' en la etapa de educación secundaria, donde el juego ya es considerado una conducta infantil, inapropiada para esta etapa (Coakley \& White, 1992; Devís-Devís, Beltrán-Carrillo \& Peiró-Velert, 2015). Por tanto, parece importante reflexionar sobre el significado de los mensajes que se transmiten a los y las jóvenes, dado que algunos de ellos pueden promover, de manera implícita e inconsciente, la disminución de la práctica de AF e incluso el abandono. La escuela y la familia deben asumir un papel nuclear en esta tarea.

El criterio de transferibilidad (Lincoln \& Guba, 1985) en este estudio se evidencia en los resultados extraídos de las experiencias, de las y los participantes en este estudio, puesto que conectan en gran medida con las ideas que se desarrollan en el campo de investigación de la psicología de la actividad física y el deporte, por lo que conviene seguir analizando desde este paradigma, las razones del cambio de referente de socialización deportiva, así como los roles de favorecedor-limitador o las implicaciones del discurso sociocultural sobre de la práctica de AF realidad.

\section{Referencias}

Abarca-Sos, A., Zaragoza, J., Generelo, E., \& Julián, J. A. (2010). Comportamientos sedentarios y patrones de actividad física en adolescentes. Revista Internacional de Medicina y Ciencias de la Actividad Física y del Deporte, 40, 410-427.

Abraldes, J.A., \& Argudo, F.M. (2009). Análisis de la motivación, participación y actitud en actividad física extraescolar. Retos: nuevas tendencias en educación física, deporte y recreación,15, 61-63.

Álvarez, E. F., López, J. C., Gómez, V., Mesa, J. B., \& Martínez, H. A. (2017). Influencia de la motivación y del flow disposicional sobre la intención de realizar actividad físico-deportiva en adolescentes de cuatro países. Retos: nuevas tendencias en educación física, deporte y recreación, 31, 46-51.

Ames, C., \& Archer, J. (1988). Achievement goals in the classroom: Students'learning strategies and motivation processes. Journal of Educational Psychology, 80, 260-267. Doi: http://dx.doi.org/ 10.1037/0022-0663.80.3.260

Amorose, A; \& Horn, T.S. (2001). Pre- to Post-Season changes in the intrinsic motivation of first year college athletes: relationships with coaching behaviour and scholarship status. Journal of Applied Sports Phychology, 13, 355-373. Doi: http://dx.doi.org/10.1080/ 104132001753226247

Biddle, S., Cury, F., Goudas, M., Sarrazin, P., Famose, J. P., \& Durand, M. (1995). Development of scales to measure perceived physical education class climate: A cross national project. British Journal of Educational Psychology, 65, 341-358. Doi: 10.1111/j.20448279.1995.tb01154.x

Biddle, S. J. H; \& Wang, C. K. J. (2003). Motivation and self-perception profiles and links with physical activity in adolescent girls. Journal of Adolescence, 26, 687-701. Doi: https://doi.org/10.1016/ j.adolescence.2003.07.003

Bolivar, A. (2004). La educación secundaria obligatoria en España. En la búsqueda de la inestable identidad. Revista Electrónica Iberoamericana sobre Calidad, Eficacia y Cambio en Educación, 2, 1-22.

Bourdieu, P. (1988). El habitus y el espacio de los estilos de vida. En: La distinción. Criterios y bases sociales del gusto. Madrid: Taurus.

Carazo-Vargas, P., \& Moncada-Jiménez, J. (2015). A meta-analysis on the effects of exercise training on the VO2 max in children and adolescents. Retos. Nuevas tendencias en Educación Física, Deporte y Recreación, 27, 184-187.

Coakley, J., \& White, A. (1992). Making decisions: Gender and sport participation among British adolescents. Sociology of Sport Journal, 9, 20-35. Doi: https://doi.org/10.1123/ssj.9.1.20

DeAndrade, A., Salguero, A., González-Boto, R., \& Márquez, S. (2006). Motives for participation in Physical activity by Brazilian adults. Perceptual and Motor Skills, 102, 358-367. Doi: 10.2466/PMS. 102.2.358-367

Deci, E. L; \& Ryan, R. M. (1985). Intrinsic motivation and selfdetermination in human behaviour. New York: Plenum.

Denzin, N. K., \& Lincoln, Y. S. (1994). Entering the field of qualitative research. En N. K. Denzin \& Y. S. Lincoln (Eds.), Handbook of qualitative research. (pp. 1-18). Thousand Oaks, CA: Sage.

Devís-Devís, J., Beltrán-Carrillo, V. J., \& Peiró-Velert, C. (2015). Exploring socio-ecological factors influencing active and inactive Spanish students in years 12 and 13. Sport, Education and Society, 20(3), 361-380. Doi: https://doi.org/10.1080/ 13573322.2012 .754753

Documento Oficial de la Generalitat Valenciana (DOGV). (2017). Resolución de 12 de julio de 2017, por la que se convocan subvenciones y asignaciones económicas a centros educativos no universitarios sostenidos con fondos públicos de la Comunitat Valenciana.

Elliot, A. J., \& Church, M. A. (1997). A hierarchical model of approach and avoidance achievement motivation. Journal of Personality and Social Psychology, 72, 218-232. 
Elliot, A. J., Gable, S. L., \& Mapes, R. R. (2006). Approach and avoidance motivation in the social domain. Personality and Social Psychology Bulletin, 32(3), 378-391. Doi: 10.1177/ 0146167205282153

Epstein, L.H., \& Roemmich, J.N. (2001). Reducing sedentary behaviour: role in modifying physical activity. Exercise and Sport Sciences Reviews 29, 103-108.

Fernández, J.G., Contreras, O.R., García, L.M., \& González, S. (2010). Autoconcepto físico según la actividad fisicodeportiva realizada y la motivación hacia ésta. Revista Latinoamericana de Psicología, 42(2), 251-263.

Garcia-Bengoechea, E., \& Strean, W.B. (2007). On the interpersonal context of adolescents' sport motivation. Psychology of Sport and Exercise, 8, 195-217. Doi: https://doi.org/10.1016/ j.psychsport.2006.03.004

Gebremariam, M. K., Totland, T. H., Andersen, L. F., Bergh, I. H., Bjelland, M., Grydeland, M., Ommundsen, I., \& Lien, N. (2012) Stability and change in screen-based sedentary behaviours and associated factors among Norwegian children in the transition between childhood and adolescence. BMC Public Health, 12, 19. Doi: $10.1186 / 1471-2458-12-104$

Gillison, F., Osborn, M., Standage, M., \& Skevington, S. (2009). Exploring the experience of introjected regulation for exercise across gender in adolescence. Psychology of Sport and Exercise, 10, 309-319. Doi: https://doi.org/10.1016/ j.psychsport.2008.10.004

Goetz, J. P., \& LeCompte, M. D. (1988). Etnografía y diseño cualitativo en investigación educativa. Madrid: Morata.

González-Cutre, D., Sicilia, A., Moreno, J.A., \& Fernández-Balboa, J.M. (2009). Dispositional flow in physical education: relationships with motivational climate, social goals, and perceived competence. Journal of Teaching in Physical Education, 28, 422-440. Doi: https://doi.org/10.1123/ jtpe.28.4.422

Green, K., Smith, A., \& Roberts, K. (2005). Young people and lifelong participation in physical education: a sociological perspective on contemporary physical education programmes in England and Wales. Leisure Studies, 24, 27-43. Doi: http://dx.doi.org/ $10.1080 / 0261436042000231637$

Haerens, L., Kirk, D., Cardon, G., De Bourdeaudhuij, I., \& Vansteenkiste, M. (2010). Motivational profiles for secondary school physical education and its relationship to the adoption of a physically active lifestyle among university students. European Physical Education Review, 16(2) 117-139. Doi: 10.1177/ 1356336X10381304

Hassandra, M., Goudas, M., \& Chroni, S. (2003). Examining factors associated with intrinsic motivation in physical education: a qualitative approach. Psychology of Sport and Exercise, 4, 211223. Doi: https://doi.org/10.1016/S1469-0292(02)00006-7

Huertas, F.J., Martínez, J.M., Tercedor, P., \& Delgado, M. (2010). El profesor de Educación Física como promotor de hábitos de vida saludables: diferentes paradigmas de promoción. En Martínez de Haro. (2010). Actividad física, salud y calidad de vida. Madrid: Ed. Fundación Estudiantes.

Janesick, V. J. (2000). La danza del diseño de la investigación cualitativa: Metáfora, metodolatría y significado. En C. A. Denman \& J. A. Haro (Eds.), Por los rincones-Antología de métodos cualitativos en la investigación social (pp. 227-251). Sonora, México: El Colegio de Sonora.

Keegan, R.J., Harwood, C.G., Spray, C.M., \& Lavallee, D.E. (2009). A qualitative investigation exploring the motivational climate in early career sports participants: Coach, parent and peer influences on sport motivation. Psychology of Sport and Exercise, 10, 361-372. Doi: https://doi.org/10.1016/j.psychsport.2008.12.003

Li, K., Iannotti, R.J., Haynie, D.L., Perlus, J.G., \& Simons-Morton, B.G. (2014). Motivation and planning as mediators of the relation between social support and physical activity among U.S. adolescents: a nationally representative study. International Journal of Behavioural Nutrition and Physical Activity, 11, 42. Doi: https://doi.org/10.1186/1479-5868-11-42
Lincoln, Y.S., \& Guba, E.G. (1985). Naturalistic inquiry. Sage: London.

Macarro, J., Romero, C., \& Torres, J. (2010). Motivos de abandono de la práctica de actividad físico-deportiva en los estudiantes de Bachillerato de la provincia de Granada. Revista de Educación, 353, 495-519.

Martínez, M. (2006). La investigación cualitativa, Revista IIPSI, 9(1), 123-146. Doi: http://dx.doi.org/10.15381/rinvp.v9i1.4033 McKercher, C., Sanderson, K., Schmidt, M. D., Otahal, P., Patton, G. C., Dwyer, T., \& Venn, A. J. (2014). Physical activity patterns and risk of depression in young adulthood: a 20-year cohort study since childhood. Social Psychiatry and Psychiatric Epidemiology, 49, 1823-1834. doi: 10.1007/s00127-014-0863-

Méndez-Giménez, A., Cecchini-Estrada, J. A., \& Fernández-Río, J. (2018). A multitheoretical approach of the students' motivational profiles in physical education: Achievement and social goals. Psicothema, 30(4), 401-407.

Menéndez, J.I., \& Fernández-Río, J. (2017). Responsabilidad social, necesidades psicológicas básicas, motivación intrínseca y metas de amistad en educación física. Retos: nuevas tendencias en educación física, deporte y recreación, 32, 134-139.

Menschik, D., Ahmed, S., Alexander, M.H., \& Blum, R.W. (2008). Adolescent Physical Activities as Predictors of Young Adult Weight. Archives of Pediatrics and Adolescent Medicine, 162(1), 29-33. Doi: 10.1001/archpediatrics.2007.14

Ministerio de Educación, Cultura y Deporte (2015). Encuesta de hábitos deportivos en España. Recuperado de: https:// www.culturaydeporte.gob.es/servicios-al-ciudadano/estadisticas/ deportes/encuesta-habitos-deportivos-en-espana.html

Moreno, J.A., \& Conde, C. (2012). Importancia del apoyo de autonomía en la figura del docente en educación física. Tándem, Didáctica de la Educación Física, 40, 17-28.

Moreno, J. E., Otero, S., Crowe, M.E., Sullivan, M. \& Rausch, B. (2013). La formación de competencias morales y sociales, prevención y/o reinserción de los sujetos con conductas antisociales. Revista Virtual de la Facultad de Psicología y Psicopedagogía de la Universidad del Salvador, 32, 56-72.

Nicholls, J.G. (1989). The Competitive Ethos and Democratic Education. Cambridge, MA. Harvard University Press.

Ntoumanis, N. (2001). A self-determination approach to the understanding of motivation in physical education. British Journal of Educational Psychology, 71, 225-242. Doi: 10.1348/ 000709901158497

Ntoumanis, N., \& Standage, M. (2009). Motivation in physical education classes: A self-determination theory perspective. Theory and Research in Education, 7, 194-202. Doi: 10.1177/ 1477878509104324

Oviedo, G., Sánchez, J., Castro, R., Calvo, M., Sevilla, J. C., Iglesias, A., \& Guerra, M. (2013). Niveles de actividad física en población adolescente: estudio de caso. Retos. Nuevas tendencias en Educación Física, Deporte y Recreación, 23, 43-47.

Patton, M. Q. (1990). Qualitative Evaluation and Research Methods (2nd ed.). Newbury Park, CA: Sage Publications.

Sparkes, A., \& Devís, J. (2007). Investigación narrativa y sus formas de análisis: una visión desde la educación física y el deporte. Educación, cuerpo y ciudad: el cuerpo en las interacciones e instituciones sociales, 43-68.

Stake, R. (2007). Investigación con estudio de casos. Madrid: Morata.

Stuntz, C. P., \& Weiss, M. R. (2009). Achievement goal orientations and motivational outcomes in youth sport: The role of social orientations. Psychology of Sport and Exercise, 10, 255-262. Doi: https://doi.org/10.1016/j.psychsport.2008.09.001

Urdan, T C, \& Maehr, M. L. (1995). Beyond a two-goal theory of motivation and achievement: A case for social goals. Review of Educational Research, 65, 213-243.

Valdemoros, M.A., Ponce, A., Sanz, E., \& Ramos, R. (2007). El valor de la salud en las experiencias de ocio físico-deportivo de jóvenes y adolescentes: conceptualización y estado de la cuestión. Contextos Educativos, 10, 117-132. 